\title{
Nuevas sonoridades en la educación musical: Voces universitarias ante la pandemia COVID-19
}

\author{
New sounds in music education: University voices in face of the COVID-19 pandemic
}

Irma Susana Carbajal-Vaca ${ }^{a}$

\begin{abstract}
:
This work presents an approach to the perceptions of students and teachers from an undergraduate music education community in the context of the COVID-19 pandemic. This exploratory study puts on the table of dialogue the understanding of the phenomenon from the own voice of its actors, to analyze the possibilities of transformation of an educational model based on closeness and personal contact that seemed not to be necessary to put in doubt. The results sketch a first real-time image of the reception of the Emerging Distance Education Program (PEED), that was implemented in a Mexican university, which will be available for further studies that can be read from the history of the present time.
\end{abstract}

Keywords:

Music education, distance learning, University education, history of the present time, cultural history of education

\section{Resumen:}

Este trabajo presenta una aproximación a las percepciones de estudiantes y maestros de una comunidad de educación musical de pregrado en el contexto de la pandemia COVID-19. Este estudio exploratorio pone sobre la mesa de diálogo la comprensión del fenómeno desde la propia voz de sus actores, para analizar las posibilidades de transformación de un modelo educativo basado en la cercanía y el contacto personal que parecía sobreentendido. Los resultados esbozan una primera imagen en tiempo real de la recepción del Programa Emergente de Educación a Distancia (PEED), que se implementó en una universid ad mexicana, la cual quedará disponible para estudios posteriores que podrán ser leídos desde la historia del tiempo presente.

\section{Palabras Clave:}

Educación musical, aprendizaje a distancia, educación universitaria, historia del tiempo presente, historia cultural de la educación

\section{Introducción}

El 14 de marzo de 2020 comenzó para la comunidad del Departamento de Música (DM) de la Universidad Autónoma de Aguascalientes, México (UAA) el periodo de confinamiento detonado por la pan demia de COVID-19. EI resguardo en las casas revolucionó un modelo educativomusical en el que la cercanía y el contacto personal eran sobreentendidos. Además de las dinámicas grupales y las clases de instrumento en sesiones uno-a-uno asentadas en el sentido común de la edu cación musical universitaria, se desarrollaban otras acciones académicas durante el año, cuya naturaleza interpersonal demandaba cercanía entre los participantes y la congregación de públicos amplios. El 20 de abril dio inicio el Programa Emergente de Educación a Distancia (PEED, 2020). Esta nueva realidad ha afectado la dinámica social de la comunidad y se reconoció como un proceso histórico inacabado, digno de ser estudiado y analizado desde la historia del tiempo presente (Carbajal-Vaca, 2020), con la cual se incorporan contenidos problemáticos y complejos ligados a sucesos recientes, que desafían los currículos prescritos (AriasGómez, 2015, p. 34).

El objetivo general de este estudio exploratorio fue documentar una primera imagen en tiempo real de la recepción del Programa Emergente de Educación a Distancia, que se implementó en el DM de la UAA para disponer de información que pueda ser leída en futuras investigaciones desde la historia del tiempo presente. Se documentó el sentir de estudiantes y profesores sobre los cambios efectuados en los últimos meses por la implementación del PEED y se analizaron implicaciones y

\footnotetext{
a Autor de Correspondencia, Universidad Autónoma de Aguascalientes, http://orcid.org/0000-0002-1575-605X. Email: susana.carbajal@edu.uaa.mx
} 
posibilidades de transformación de un modelo educativo que se percibía sobreentendido.

De acuerdo con Fazio-Vengoa (1998) la historia del tiempo presente se interpreta y se escribe en la inmediatez de una coyuntura ubicada dentro de un proceso que se reconoce como un posible cambio histórico. El propósito es comprender la significación del acontecimiento para establecer el vínculo que existe entre pasado y presente. La historia del tiempo presente permite recuperar la voz de protagonistas que estén en condiciones de relatar su testimonio. Se requiere la existencia de una memoria social viva, disponer de procesos históricos en desarrollo que puedan serconsiderados como objeto de estudio y la detección de fenómenos no muy lejanos que hayan afectado la dinámica de las sociedades (Arias-Gómez, 2015: Franco y Levín, 2007; López, Figueroa y Rajland, 2010). Frente a la historiografía tradicional que se caracteriza por el análisis de situaciones históricas concluidas, la historia del presente se aboca a la dificultad de analizar situaciones inacabadas y posibilita el análisis de la realidad social vigente al poneren relación la historia vivida, su escritura, los actores, los testigos de la historia y los historiadores (Aguirre-Lora, 2016; ArósteguiSánchez, 2004; Soto-Gamboa, 2004). Dada la inmediatez de los sucesos, existe el riesgo de desvelar dificultades ético-políticas, porque la cercanía despierta pasiones en las que el historiador podría verse involucrado (AriasGómez, 2015; López, Figueroa y Rajland, 2010).

Las licenciaturas en música en el país operan a partir de ciertas tradiciones y han puesto en marcha acciones académicas específicas que las caracterizan como sistemas culturalesque los diferencian entre sí. Se asume que estas acciones pueden ser resultado de la conciencia intencional de sus actores, cuyos significados pueden ser recuperados mediante un tratamiento etnometodológico (Schütz y Garfinkel en De la Garza-Toledo y Leyva, 2012; Garfinkel, 2006).

El 6 de mayo se enviaron cuestionarios semiestructurados a profesores y estudiantes mediante una aplicación digital que ha estado disponible desde hace varios años para la comunidad académica, pero que, en este momento, se desveló como un recurso emergente para los investigadores. Al 8 de junio de 2020 se recibieron respuestas de 64 estudiantes (16 de segundo semestre, 16 de cuarto, 10 de sexto, 14 de octavo y 8 de décimo), de una población de 93, y 17 profesores, de una plantilla de 32. Las respuestas fueron anónimas, por lo cual, el riesgo de sesgar la información, latente en esta perspectiva, disminuyó considerablemente. Las respuestas de la comunidad se incluyeron en el texto con las siglas CP (Cuestionario Profesor) y CE (Cuestionario Estudiante), seguidas del número de cuestionario aplicado. Se organizaron las voces de la comunidad en 4 categorías de análisis: (1) actitud ante el cambio, (2) retos de la nueva realidad, (3) preocupaciones y nuevas perspectivas, (4) resistencias y áreas de oportunidad, las cuales esbozan una primera imagen en tiempo real de la recepción del PEED (2020) en el DM de la UAA.

El primer apartado corresponde al análisis de acuerdo con las categorías señaladas. Posteriormente se analizan las implicaciones y posibilidades reales hacia el rediseño de un programa educativo acorde con el cambio social que podría derivarse de la coyuntura del COVID-1 y se cierra con un espacio para conclusiones.

\section{Percepción del PEED}

\section{Actitud ante el cambio}

Se aprecia que los profesores identifican en las acciones académicas una orientación hacia la formación integral y la intencionalidad humanista del modelo educativo:

La UAA concibe la educación como un proceso intencional y sistemático, cuya finalidad es la formación integral de la persona, esto es, el desarrollo pleno de todas sus facultades, posibilitando así la construcción de una sociedad más humana en la que se vivan valores como el respeto, la libertad, la tolerancia, la igualdad de derechos de todos los hombres, la paz, el espíritu de servicio, la honestidad, el liderazgo y la actitud positiva hacia el cambio y la excelencia (MEI, 2006, p. 4).

Ante la aplicación del PEED (2020), los 17 profesores que respondieron el cuestionario muestran en sus comentarios una actitud positiva y solidaria hacia el cambio y creen estar haciendo su mejor esfuerzo para lograr los objetivos de los programas de materia:

$\mathrm{Me}$ he propuesto estar disponible para mis estudiantes todo el tiempo y asegurarme de que ellos entiendan que todo lo que se hace es para el bien de ellos, para su aprendizaje (CP-2).

Creo que sí se extraña el contacto personal, pero las metas de aprendizaje se han cumplido (CP-13).

Los comentarios de los estudiantes muestran que se percibe esta actitud positiva:

[...] los maestros están muy comprometidos con su trabajo de enseñarnos, y nos han dado herramientas muy buenas que nos han ayudado bastante; [...] siempre están en la disposición de ayudarnos; son pocos los maestros que no nos han dado materiales que nos ayuden a aprender realmente (CE-8).

Nuestra licenciatura actualmente enfrenta un gran reto con la modalidad a distancia de clases que son sumamente prácticas y requieren la intervención directa de un profesor para la realización de observaciones y correcciones pertinentes, sobre todo en las materias de instrumento principal y música de cámara. No es mi caso pero, sin duda, están en esta misma situación las materias de solfeo y armonía. Deben de ser sumamente complicadas para los semestres que actualmente las cursan en esta modalidad en línea (CE-43). 
Se encontraron expresiones emotivas, envueltas en un halo de nostalgia por las prácticas acostumbradas:

Nada puede sustituir el momento sustancial y sensible que surge en nuestro arte sonoro, ese instante que involucra todos nuestros sentidos y aflora nuestros sentimientos (CP-12).

Esperamos volver lo más pronto posible para tener las clases normales (CE-1).

Ojalá se resuelva pronto esta contingencia para regresar a clases presenciales (CE-3).

Ya quiero volver (CE-28).

Varias de las respuestas de los estudiantes muestran una actitud solidaria y esperanzadora ante los retos que han tenido que asumir los profesores:

Es un poco complicado cumplir con todas las expectativas para el trabajo; eso genera un poco de estrés porque es una forma de trabajo que también es nueva para los profesores (CE-4).

Creo, que estudiar en línea es mucho más difícil, pero no imposible, es cuestión de costumbre y de poco a poco encontrar mejores métodos, para volver la clase mas efectiva (CE-11).

Todo esta bien; al principio sí era algo extraño pero ya me acostumbré (CE-14).

Es un poco difícil adaptarse porque fue algo repentino, pero con el tiempo espero las cosas mejoren (CE-14).

A pesar de esta situación que estoy pasando, ha sido una buena experiencia el estar estudiando de esta manera; ha complicado varias cosas, pero siempre busco la manera de dar el mejor resultado (CE-29).

Es bastante complicado tomar clases virtuales, pero creo que es porque no estábamos preparados para esta situación (CE-38).

Es significativo que los comentarios normalmente se presentaran en equilibrio con la contraparte de oportunidad de apertura a nuevos aprendizajes:

Este momento de distanciamiento social ha propiciado más interés por las plataformas virtuales, con ello hemos logrado enriquecer nuestra labor desde otro punto de vista (CP-12).

Realmente me ha motivado a ser mejor maestro. Cada clase que preparo, debo reflexionar con mucho cuidado sobre la manera de transmitir los contenidos para hacerlo con eficacia y eficiencia (CP-2).

He aprendido muchas herramientas para la enseñanza a distancia, ha sido muy enriquecedor (CP-7).

Se encontraron respuestas que señalan que las decisiones que tomó la universidad y los recursos que puso a disposición de los profesores ante la emergencia fueron acertados:

Las medidas que ha tomado la Universidad me parecen adecuadas, el aula virtual funciona generalmente muy bien y los alumnos han respondido muy bien ante la contingencia (CP-9).
De cierta manera la Universidad a distancia está funcionando (CP-1).

\section{Retos de la nueva realidad}

Aunada a la percepción general, las respuestas evidencian que los recursos tecnológicos dispuestos no tienen la misma funcionalidad para todas las asignaturas. Dado que en el modelo educativo-musical la cercanía era sobreentendida, los docentes han tenido que asumir algunos retos importantes:

Hay ciertas dificultades para llevar a cabo una clase tan práctica y de conjunto como música de cámara (CP-1).

Es un poco difícil trabajar a distancia, sobre todo en el instrumento y música de cámara, ya que no se pueden trabajar todos los aspectos técnicos (CP-5). Mi experiencia como docente en esta situación me ha resultado dificil, la clase de instrumento es presencial, por lo tanto se pierde demasiada información y tiempo cuando se hace a través de video-llamada, además de que el audio no es lo mismo que en vivo (CP-16).

A pesar de la capacitación y recursos que la institución puso a disposición de los profesores, la música tiene especificidades que no pueden ser atendidas en plataformas genéricas, como el Aula Virtual, por lo que algunos profesores han tenido que auxiliarse en otros medios:

No es posible realizar a plenitud un trabajo de revisión musical en la plataforma de Aula Virtual, así que tuve que apoyarme en [...] otras plataformas y capacitarme sobre la marcha en ello. Aun cuando, previamente, tomé un curso pero, éste no nos orienta a los docentes de música acerca de cómo resulta más práctico y viable hacer la revisión a alumnos por estos medios [...] El Aula Virtual requiere mayor capacidad y los docentes más capacitación específica en recursos para grabar videos de alumnos de música y en el uso de plataformas que nos faciliten nuestro trabajo (CP-8).

Esta perspectiva se confirma también en las voces de los estudiantes al valorar la eficiencia de las asignaturas que cursan actualmente.

La comunicación con los estudiantes se presentó como uno de los grandes retos:

Los profesores hemos tenido que ser creativos para mantener comunicación con los estudiantes y hemos tenido que dedicar tiempo a conocer las herramientas que la universidad nos ha puesto a disposición (CP-13).

34 de los 64 estudiantes que respondieron el cuestionario reportaron no haber tenido ninguna dificultad de comunicación con los profesores. El resto de ellos señaló que las dificultades se relacionaban a deficiencias en la conectividad en casa o equipo inadecuado.

Algunos profesores generaron grupos en WhatsApp, utilizaron la plataforma de Zoom, Microsoft Teams o 
Google Meet, sin embargo, la diversificación no fue garantía de comunicación porque algunos estudiantes perdieron su empleo y no disponían de recursos para contratar el servicio de internet en casa o cargar datos en su teléfono celular.

Cabe señalar que, como parte de este programa emergente, la universidad, además de las becas y la prórroga de pagos, puso a disposición de estudiantes en condición de vulnerabilidad económica equipo de cómputo y conexión a internet mediante el Programa Institucional de Accesibilidad a Tecnologías de la Información y la Comunicación. Se ha prestado equipo a 350 estudiantes y se ha dado acceso a internet alrededor de 300 (PEED, 2020). 21 estu diantes de la licenciatura en música respondieron haber solicitado algún tipo de apoyo a la universidad.

[...] el apoyo que solicité fue el de préstamo de equipo. Fue muy frustrante para mí que no pude realizar las actividades como indicaban los maestros los primeros días de clases virtuales, ya que muchas dudas se realizaban por zoom, como se les indicó, y yo no contaba con esa aplicación, porque no contaba con un equipo con las características que se requiere para instalarse. Me fue muy grato que los maestros, algunos de inmediato, y otros poco a poco, fueran comprendiendo esto que nos afectaba a pocos compañeros, por lo que buscaron varias formas de comunicación (CE-54).

Este reto de comunicación no ha sido únicamente paralos profesores, sino entre los propios estudiantes. La tutora longitudinal ha mantenido comunicación con todos los estudiantes a través de los cinco jefes de grupo, quienes también han tenido que asumirnuevas responsabilidades. Creo que todos los profesores han hecho un esfuerzo maravilloso para aprovechar al máximo las herramientas que se tienen. Por mi parte, a veces sí hay momentos en que no me siento muy motivada o estable por problemas con mi equipo de cómputo y porque creo que podríamos aprender más y aprovechar mejor las clases si fueran presenciales; pero, en general, creo que todo está en orden. Intentaré estar más atenta a los avisos de la jefa de grupo porque ella también ha hecho un esfuerzo increíble (CE-35).

\section{Preocupaciones y nuevas perspectivas}

Frases como "que los alumnos sigan estudiando" (CP-6), revelan la preocupación de los profesores por la interrupción de procesos de práctica que son indispensables para el desarrollo instrumental. Al interior de las academias, los profesores están valorando más posibilidades para apoyar a los estudiantes:

En varias Academias está surgiendo la propuesta de crear programas de regularización y cursos de inducción para los alumnos, cosa que me parece pertinente debido a la situación (CP-1).
Creo que esta situación nos hizo dar cuenta, tanto a nosotros como alumnos, como a los profesores, de qué tipo de materias podrían darse en modalidad a distancia, esto beneficiando el tiempo de clase para otro tipo de actividad (CE-20).

Algunas materias han perdido bastante calidad dada la repentina modalidad a distancia; sin embargo, con el estudio autónomo y la disposición de los profesores, que siempre ha estado, hemos podido mantenernos al tanto y no perder el conocimiento adquirido antes del inesperado confinamiento (CE32).

Mis profesores se han mostrado abiertos y comprensivos frente a la situación. Veo que se ha hecho un esfuerzo por parte de ellos y mis compañeros por hacer las cosas de la mejor manera. A pesar de las deficiencias y problemas, creo que por mi parte he sido más autocrítico con mi manera de aprender, $y$ he mejorado mis habilidades para adquirir conocimiento de manera autodidacta que, personalmente, considero ya era una de mis fortalezas (CE-21).

Se ha hecho lo mejor posible tanto de parte de los maestros como de nosotros; es difícil para todos trabajar así cuando no se estaba acostumbrado, ha sido muy pesado pues las exigencias tanto para los maestros como para nosotros creo que han sido demasiadas, pues se intenta tratar el programa del semestre como si fuera presencial y creo que debido a las condiciones hay que adaptarse y disminuir cierta carga; en lo personal termino agotada, harta y cansada de estar frente a la computadora tanto tiempo, pero en general los maestros se han portado muy comprensivos y se están esforzando por hacer lo mejor que se pueda. Nuestra carrera es muy práctica, así que se ha hecho lo mejor que se puede por ese lado (CE-22).

Esta nueva situación también ha causado incertidumbre en los estudiantes, sobre todo respecto del nivel y la calidad que podrán alcanzar este semestre. Aunque los comentarios se presentaron casi siempre en equilibrio con percepciones positivas, los que incluyen palabras que definen emociones poco favorables son recurrentes.

La contingencia ha resultado muy difícil, ya que la ansiedad afecta significativamente mi desempeño, pero ha resultado también benefactora para poder desarrollar la paciencia, el trabajo en equipo y la organización (CE-51).

[...] no sé como sentirme al respecto, a veces me siento estable y motivada, pero conforme han pasado las semanas sí me siento un poco cansada y desmotivada porque siento que estoy más tiempo en la computadora que en mi instrumento; no siento un balance como tal porque me siento presionada por tener en mente las entregas que tengo que hacer durante la semana en línea. Aunque debo de decir que también la presión a veces me ayuda a sacar mejor las cosas, pero no sé, siento que nunca voy a acabar. Son sentimientos encontrados ya que sí extraño tener clases presenciales, escuchar a los compañeros y a los maestros, pero también de cierta manera esta situación me hace despertar, a practicar la disciplina aún más viva en mi vida (CE26). 
Lo único que me gustaría comentar es que la semana pasada me sentí algo desmotivada. A pesar de que hacemos lo mejor que podemos para aprovechar las clases, nunca será igual a tener clase de violín presencial. He notado que en estos meses he estudiado menos y he tenido un menor rendimiento de estudio, y me preocupa no crecer musicalmente en este semestre; sobre todo porque el siguiente año estará destinado a mi repertorio de graduación, y me pone triste no alcanzar el nivel que se espera para ello (CE-45).

Me he sentido un poco decepcionado por no tener un avance en las materias prácticas en linea como lo hacia presencialmente; [...] entiendo que es contingencia y confinamiento, por lo que me he adaptado muy bien y he logrado más o menos tener ese avance; sin embargo no es el mismo (CE-55).

Se reportaron problemas de conectividad por pérdida de señal hasta por tres días, falta de equipo de cómputo adecuado, falta de instrumento y, principalmente, la insuficiencia del Aula Virtual para desarrollar las actividades que requiere la licenciatura en mú sica. El Aula Virtual es uno de los espacios de trabajo institucional disponible para estudiantes y profesores, el cual está hospedado en la plataforma Moodle (Ámbito Académico, 2020). Los señalamientos van desde dificultades simples para el registro de asistencia, hasta la inconformidad de los profesores por la inefectividad de la plataforma para el almacenamiento de video; comentarios que quizá fueron motivados más por la inexperiencia y desconocimiento de las herramientas que por la resistencia a la modalidad virtual:

Quizá la poca costumbre que tienen los alumnos a revisar el Aula Virtual y, de igual manera, los profesores de no usarla como una herramienta más (CP-3).

Sigue siendo confuso lo de la asistencia por medio del aula virtual, sobre todo cuando no tenemos clase y el día se dedica a dudas que hayan surgido (en mi caso he tenido sólo una, y la hice comunicar). En caso de no tener dudas sigo sin saber si tengo que permanecer conectada todo el tiempo o si no hace falta. Por esto mismo tengo dudas si he tenido alguna falta por no estar conectada toda la sesión (CE-10).

Algunos profesores comenzaron a visualizar distintos escenarios posibles y necesidades que sería urgente atender:

El uso de las plataformas y redes como herramientas de enseñanza-aprendizaje. Para los Profesores la actualización del uso de las nuevas tecnoogías (CP-11).

Hay que plantear la posibilidad de reestructurar las clases grupales e individuales de tal forma que sea posible complementar las clases de manera virtual. Que las plataformas que ofrece la universidad como el Aula Virtual tengan la posibilidad de hacer videoconferencias para poder tener clases individuales o grupales (CP-15).
[...] enseñanza de estrategias de estudios musicales con el apoyo de las nuevas tecnologías de la información, a las cuales el alumnado no está habituado; profundización de la consciencia en el alumnado acerca de la misión del músico en la sociedad, la cual depende del escenario donde se concretice (CP-10).

Habría que valorar cuáles asignaturas podrían permanecer a distancia para optimizar los espacios del Departamento de Música. Los ensambles vocales e instrumentales son los que más han sufrido (CP-13).

Creo que las asignaturas de instrumento principal se pueden seguir impartiendo de forma presencial, ya que son solo dos personas en un salón donde se puede guardar perfectamente la sana distancia y respetar todos los protocolos de salud (CP-14).

\section{Resistencias y áreas de oportunidad}

No hubo comentarios que descalificaran el trabajo de los profesores; sin embargo, sí se registraron respuestas que confirman que el modelo educativo-musical presencial era sobreentendido, por lo que algunos comentarios podrían interpretarse como resistencia:

La escuela virtual no funciona y menos en una carrera como ésta (CE-23).

Creo que la carrera de música funciona mejor en clases presenciales ya que la mayoría de las materias son prácticas (CE-18).

Aunque suene reiterativo: toda materia, aunque sea teórico-práctica o sólo práctica y más hablando de la materia de música, requiere enseñanza presencial, al menos para el nivel que se supone estamos cursando (licenciatura); [...] si se hablara de un taller en linea, serían entendibles sus límites (CE-34).

Otras opiniones revelan disgusto, frustración o resignación ante las dificultades que han tenido para adaptarse a la nueva situación:

Hacer lo que se puede con lo que se tiene no logra los objetivos planteados, sólo cubre los requisitos (CE-15).

Las clases de coro no se han llevado como me gustarían (CE-7).

No he estoy teniendo muchas dificultades pero hay materias que definitivamente no son aptas para este tipo de clases. Pero pues, no hay muchas alternativas (CE-25).

Es una situación en la cual nos vemos todos "obligados" a participar de esta manera por la situación actual de salud. Puede ser difícil en momentos; sin embargo hay que cumplir con lo que nos gusta (CE-57).

Me gustan más las clases presenciales, se me hacen más dinámicas y divertidas, [...] siento que se están perdiendo muchas cosas importantes. La verdad hubiera preferido que nos dejaran iniciar nuevamente el semestre para poder absorber todo el material de una buena manera (CE-8).

Me cuesta mucho trabajo estudiar en casa, psicológicamente siempre he visto mi hogar como un lugar de descanso al que llego después de un día de estudio y donde puedo liberarme del estrés, 
ahora tengo estrés en mi lugar de descanso. Además hay muchos distractores que complican el estudio en casa (CE-16).

Los estudiantes señalaron con claridad lo que podría mejorarse en las nuevas estrategias:

Es una situación extraordinaria no prevista. El internet y los medios pueden ser lentos o fallar. Debería hacerse lo necesario y no saturar de información que no está siendo comprendida por todos al 100\% (CE-59).

En más de una materia, el tiempo necesario para la realización de una actividad consume una gran cantidad de horas semanales, lo que perjudica el estudio del instrumento, por ejemplo. Tal vez deberían considerar bajar un poco la expectativa en la exigencia a la cual estamos acostumbrados, ya que hay muchos factores que perjudican nuestro desempeño; sólo por mencionar alguno: el estado de ánimo tan tenso que la mayoria experimenta. Aplaudo a aquellos maestros cuya labor sigue, a aquellos que se esfuerzan en crear actividades entretenidas, con calidad y que nos alegran el día, deberían ser ejemplo para aquellos que atiborran de actividades sin chiste como entregar apuntes, ver videos de youtube de más de una hora y sin contacto cercano (CE-20).

Creo que es un error que se piense que por estar en casa se tiene más tiempo y por tanto se puede dejar más trabajo. Nuestro cerebro no está recibiendo los estímulos a los que está acostumbrado, por lo que estar en casa tanto tiempo puede ser muy estresante. Hay quienes viven solos y es aún más difícil. En suma, esto debería ser una cosa importante que los profesores deben considerar: más no es mejor siempre (CE-37).

A pesar de la visión crítica anterior, las situaciones que reportan los estudiantes como dificultad están más relacionadas con deficiencias tecnológicas, problemas económicos y con situaciones personales particulares, que con el desarrollo de las clases y el desempeño de los profesores:

No me es muy conveniente pasar mas de una o dos horas frente al computador, ya que soy fotosensible y me daña la vista. Otro problema que trato de manejar, es que aquí en mi casa, por el momento sólo contamos con un equipo de cómputo y en varias ocasiones se nos juntan las clases a mi hermana y a mí; [...] sin embargo he tratado de ir avanzando en las tareas poco a poco, y me mantengo al corriente (CE-46).

\section{Nuevas sonoridades}

Algunos estudiantes lograron reflexiones que revelan una comprensión profunda del modelo educativo; expresaron claramente el valor que le han dado ahora al aprendizaje autónomo, generaron argumentos que tienen que ver con la optimización del tiempo y fueron críticos con lo que consideran sobrecarga de trabajo. También pusieron sobre la mesa condiciones actuales que incluso consideran mejores, lo cual da pie a refleixionar sobre cambios y adecuaciones al currículo actual:

De manera personal, se me hacen muy bien las clases en linea. La verdad a mí se me hacen mejor porque [...] el transporte público no pasa tan seguido $y$ tardo mucho en llegar (CE-31).

Me parece muy bien esta estrategia de la Licenciatura en línea ya que considero que en la gran mayoría de las materias sí se puede avanzar (CE-2).

Es una buena oportunidad para pensar en qué se le ofrecerá a los alumnos que pierden este semestre clases maestras, talleres, cursos, uso de las instalaciones para los egresados, etc. (CE-15).

Ha sido una situación bastante difícil por [...] el encierro, lo que me ha causado bastante estrés en algunos momentos. Lo bueno es que se está convirtiendo en una suerte de autoaprendizaje bastante acelerado y de autodisciplina que me obliga estar más al pendiente de mis responsabilidades académicas (CE-61).

Se solicitó a los estudiantes que mencionaran cuáles eran las asignaturas que consideraban que habían sido desarrolladas de manera más efectiva y cuáles no lo habían logrado de manera óptima. Más que localizar asignaturas en particular, el interés central fue recuperar opiniones y descripciones de prácticas que permitieran reconocer valoraciones específicas sobre las estrategias que las hacen efectivas:

Me está ayudando bastante ya que por medio de un chat grupal todos mis compañeros y yo nos mandamos los ejercicios del día y así podemos retroalimentarnos (CE-2).

La maestra nos envía claramente qué hay que hacer y nos da un margen adecuado para realizar la actividad, nos asesora si es necesario y nos deja administrar nuestro tiempo (CE-22).

Respuestas como éstas sugieren que acciones que promueven la comunicación entre los compañeros, la libertad y la autonomía son valoradas positivamente por los estudiantes. Las percepciones generales tanto de profesores como de estudiantes se orientaron a la categorización de las asignaturas -teóricas, prácticas y teórico-prácticas-. A partir de ellas señalaron las asignaturas prácticas como las más afectadas por la forma de trabajo a distancia y las teóricas, como las mejor implementadas en esta modalidad:

Las materias más efectivas han sido aquellas que no son prácticas, ya que es sencillo dejar las instrucciones escritas y hacer los trabajos que se encargan (CE-12).

Por su naturaleza teórica, es más sencillo adaptarlas a esta modalidad (CE-21).

Los estudiantes mencionaron que algunas materias, aun siendo presenciales, ya se desarrollaban en plataformas digitales, por lo que la pandemia no afectó la dinámica de trabajo. A pesar de esta apreciación diferenciada sobre la 
naturaleza teórica y práctica, en las respuestas específicas sobre la efectividad de las asignaturas se encontró que son las estrategias implementadas por los profesores las que determinan la percepción de efectividad y no la naturaleza sobreentendida. Así, en asignaturas como Solfeo, cuya naturaleza es práctica por las actividades desarrolladas en aula en un modelo tradicional, los estudiantes valoraron más la organización, el orden, el envío de audios para el entrenamiento auditivo, los materiales disponibles en línea y la comunicación (CE-25, 26, 49, 56, 57):

El maestro ha sido bastante claro en la forma que debemos trabajar en nuestras casas (CE-10).

En las clases de instrumento, también de naturaleza práctica, valoraron la estrategia de envío de videos y las videoconferencias como favorables:

Enviamos videos a la maestra y ella nos da retroalimentación de algunas cosas que se pueden corregir (CE-3).

Piano Complementario, porque las clases las tomo por Zoom y es casi igual que cuando iba a clase normal aunque, obviamente, prefiero que sea en vivo (CE-18).

Instrumento Principal y Piano Complementario porque las clases por videollamada resuelven todas las dudas (CE-28).

Piano, ya que está muy bien el que cada semana estemos entregando videos de lo que trabajamos en la semana (CE-31).

Investigaciones realizadas en esta comunidad académica han señalado que estrategias apoyadas en tecnologías de información y comunicación para la práctica instrumental -como el portafolio electrónico, en el que los estudiantes muestran a sus profesores lo que ocurre en su práctica individual en casa- son estrategias metacognitivas efectivas (Capistrán-Gracia, 2018). Las respuestas de los estudiantes a este cuestionario son quizá las primeras evidencias empíricas en el contexto de esta comunidad que lo confirman.

Habrá que tomar en consideración que los resultados de estas nuevas estrategias de práctica ya son visibles en las participaciones de los estudiantes en el proyecto "El arte en tiempos del coronavirus"; iniciativa de la doctora Raquel Mercado Salas, impulsada por el Centro de las Artes y la Cultura (CAyC) y puesto en línea, con la colaboración del maestro Marco Antonio Marmolejo, desde el 18 de mayo de 2020 en el Canal Youtube del $\mathrm{CAyC}^{*}$. En este canal se transmiten cápsulas de profesores, estudiantes y colaboradores externos, las cuales han realizado desde sus casas para mostrar los logros alcanzados durante este tiempo de confinamiento:

Se trata primordialmente de difundir el objetivo del programa a través de los resultados que vamos obteniendo (Comunicación vía WhatsApp con Carlos Ávila Aréchiga, 09/06/2020).

La idea central fue abrir un canal de comunicación para dar a conocer cómo están participando las artes en este tiempo de confinamiento $y$

cómo desde nuestra experiencia con el arte, ya sea como espectadores o como productores, algunos podían no sólo hablar de la crisis, sino hacer algo al respecto en el sentido emocional [...] configurar experiencias sensibles a partir del arte en todas sus dimensiones (Comunicación vía WhatsApp con Raquel Mercado Salas, 09/06/2020).

Aunque este proyecto es originalmente para la difusión del trabajo universitario, en las participaciones se evidencia un avance real en la ejecución instrumental que, muy probablemente, está relacionado con el nivel de confianza alcanzado con la utilización de tecnología durante el semestre.

Otras de las materias eminentemente prácticas son Dirección Orquestal y Conjuntos de Cámara. También en ellas se sobrepuso el valor de la estrategia didáctica al de su naturaleza:

Los temas son bien trabajados y se presta para la evaluación [...] El conocimiento inicial es suficiente para poder echar a andar por cuenta propia el trabajo (CE-4).

Los trabajos que dejan no ocupan demasiado tiempo, y dejan material que está bien explicado (CE-6).

Dirección orquestal, aunque es una materia totalmente presencial, el maestro [...] ha desarrollado una estrategia más que efectiva, usando sus conocimientos y creatividad a través de las herramientas electrónicas que ofrece la plataforma de Facebook. Cabe resaltar la gran labor realizada por el maestro al no ceñirse a las pobres e insuficientes estrategias adoptadas por el departamento (CE-15).

La visión crítica de los estudiantes es bienvenida; sin embargo, algunos de los comentarios también podrían ponerse en la mesa de diálogo para analizar si se está configurando una comprensión distorsionada sobre las metas de aprendizaje:

Las materias más efectivas han sido aquellas que no son prácticas, ya que es sencillo dejar las instrucciones escritas y hacer los trabajos que se encargan (CE-12).

Creo que con la única materia que realmente no hay problema es con historia, porque la información no se tiene que poner en práctica, sólo se tiene que memorizar, aparte, el profe [...] se ha esforzado mucho en sus clases en línea (CE-11).

Esta visión instructiva es uno de los riesgos de utilizar sistemas como Moodle que son sumamente estructurados. Aunque existe libertad de utilización, la

* https://www.youtube.com/channel/UCQpinFsKDw6vnx8Q61WxaZA 
naturaleza de la plataforma es instruccional, lo que podría propiciar que sólo se implemente como un gestor de actividades para contabilizar el cumplimiento.

Habría que señalar que, precisamente las materias de historia de la música han despertado un interés especial de los profesores de esta comunidad. La estrategia ideada e implementada por el maestro Juan Pablo Correa cuando fue coordinador de la Academia de Cultura, Tecnología y Educación Musicales fue que los estudiantes y profesores de estas asignaturas produjeran programas de radio dirigidos al público en general. El programa Ventana al Sonido, transmitido semanalmente por Radio UAA, fue concebido como una estrategia de aprendizaje situado para disminuir el riesgo que llevan estas asignaturas de desarrollar dinámicas de aprendizaje memorístico.

\section{Conclusiones}

Las medidas sanitarias implementadas en la comunidad académica estudiada y la nueva dinámica de trabajo podrían hacer plausibles transformaciones que, además de ajustarse al cambio social derivado de la pandemia, atiendan problemáticas de movilidad y espacio que ya habían sido señaladas mucho antes del confinamiento.

Se logró recuperar el sentir de la comunidad. Las voces de los estudiantes reflejan la manera como han recibido las estrategias de sus profesores. No se presentó ni una aceptación, ni un rechazo generalizados hacia las nuevas prácticas. Las respuestas fueron diversas, pero queda de manifiesto en esta exploración que los estudiantes emiten sus juicios ensayando aspectos desfavorables contrastándolos con los favorables. Los comentarios de profesores revelan afinidad y conocimiento del modelo educativo. Se reconoce flexibilidad y disposición a modificar las prácticas docentes sobreentendidas.

Es evidente el respeto y solidaridad mutuos entre estudiantes y profesores. No se encontró ninguna opinión que demeritara el trabajo realizado por la comunidad universitaria en su conjunto. Incluso ante la solicitud expresa de señalar las asignaturas menos efectivas, las respuestas aparecieron en equilibrio con valoraciones positivas.

Algunas de las apreciaciones desfavorables no se relacionan directamente con la situación de confinamiento, sino con el desempeño general de la asignatura por carencia de equipo especializado disponible en la universidad y que, por ahora no puede ser utilizado por los estu diantes. Por ejemplo, alumnos que no tienen teclado en casa, practicaban en la escuela. La universidad puso a disposición el préstamo de computadoras, pero la carrera de música requiere equipo especializado e instrumentos que no deben salir del departamento.
La percepción de los estudiantes y las estrategias implementadas por los profesores muestran que la clasificación de las asignaturas por su naturaleza teórica o práctica no es el factor decisivo para ligar su efectividad a la modalidad presencial. Asignaturas que normalmente se conciben como eminentemente prácticas, han podido desarrollarse de manera exitosa en la modalidad a distancia. Parece ser que la creatividad de los profesores para incluir distintas herramientas en las estrategias es el factor más apreciado por los estudiantes.

Se reconocen como acciones necesarias:

La implementación de cursos de capacitación para profesores y estudiantes en los que se incluyan, además de el uso de tecnología, temáticas para comprender de manera más amplia la importancia del estudio autónomo, el diseño de estrategias de aprendizaje autodidacta y la conformación del sentido común como proceso para acostumbrarse a la nueva realidad.

Tener presente en el diseño de estrategias las problemáticas derivadas de la sobreexposición en las plataformas virtuales, la complejidad y tiempo que requiere cada una de las actividades, el cansancio, el estrés y la sobreestimulación visual.

Considerar en las planeaciones las experiencias de los estudiantes respecto de las estrategias que les han resultado efectivas, como las videoconferencias, el envío de videos y la exposición de sus trabajos en canales públicos y reforzar las actitudes que esta comunidad ha señalado como guía en este tiempo de contingencia: la paciencia, el autoaprendizaje, el trabajo en equipo, la autocrítica y la comunicación.

La implementación de un modelo a distancia para la licenciatura en música, en esta primera imagen parece plausible; sin embargo, habrá que pensar que tanto profesores como estudiantes deben disponer de tecnología actualizada y estar en condiciones de solventar costos de conectividad, adicionales a las cuotas de la universidad.

Explorar y documentar desde el inicio las transformaciones en las prácticas educativas por efecto de un suceso extraordinario en la historia presente, permitirá contrastar experiencias futuras y valorar si pueden ser consideradas como el inicio de un cambio social. El COVID-19 ha afectado las sociedades mundialmente y ha dejado expuestas una gama de diferencias que nos obligan a pensar en nuevas estrategias para lograr las metas de equidad e inclusión. Queda de manifiesto que se requerirá la capacidad creativa de toda la comunidad.

\section{Referencias}

[1] Aguirre-Lora, M.-E. y Márquez-Carrillo, J. (2016). Historia e historiografía de la educación en México, 2002-2011: vicisitudes de un territorio abierto. En M. E. Aguirre-Lora. Historia e historiografía de la 
educación en México: hacia un balance, 2002-2011, I. 35-60. Ciudad de México: Anuies / Consejo Mexicano de Investiga ción Educativa.

[2] Ámbito Académico (2020). Plataforma Virtual Moodle. Universidad Autónoma de Aguascalientes. Recuperado de: https://ambitoacademico.uaa.mx

[3] Arias-Gómez, D. H. (2015). La enseñanza de la historia reciente y la formación moral. Dilemas de un vínculo imprescindible. En Folios, Segunda época 42, pp. 29-41. Recuperado de: http://www.scielo.org.co/pdf/folios/n42/n42a03.pdf

[4] Aróstegui-Sánchez, J. (2004). La historia del presente. ¿Una cuestión de método? En Navajas Zubeldia, C. (ed.), Actas de IV Simposio de Historia Actual. pp. 17-19 de octubre de 2002. Logroño: Gobierno de La Rioja. Instituto de Estudios Riojanos, pp. 41-75. Recuperado de: $\underline{\text { https://dialnet.unirioja.es/servlet/articulo?codigo }=1036594}$

[5] Capistrán-Gracia, R. W. (2018). El portafolio electrónico como instrumento de evaluación y como estrategia de autorregulación en la formación de profesionales en música. En Revista Educación, 42(2). Universidad de Costa Rica. DOI: https://doi.org/10.15517/revedu.v42i2.25937

[6] Carbajal-Vaca, I-S. (2020), Implicaciones teórico-metodológicas en la historia presente de la educación musical de nivel superior en México, Revista Iberoamericana de Educación Superior (ries), 9(32), pp. 133$147 . \quad$ Recuperado de: https://www.ries.universia.unam.mx/index.php/ries/article/view/818/12 $\underline{85}$

[7] De la Garza-Toledo, E. \& Leyva, G. (2012). Tratado de Metodología de las Ciencias Sociales: Perspectivas actuales. Ciudad de México: Fondo de Cultura Económica.

[8] Fazio-Vengoa, H. (1998). La historia del tiempo presente: una historia en construcción. Historia Crítica, 17, pp. 47-57. Bogotá: Universidad de Los Andes. Recuperado de: https://www.redalyc.org/pdf/811/81111329004.pdf

[9] Franco, M. \& Levín, F. (2007). El pasado cercano en clave historiográfica. En Franco, M. \& Levín, F. (Comp.). Perspectivas y desafíos para un campo en construcción. Buenos Aires: Paidós. Recuperado de: http://files.pluriversidadoikologias.webnode.es/2000000192c6502d5e4/2013\%20CAPACITACION\%20PRSENCIAL\%20FRAN CO\%20LEVIN.pdf

[10] Garfinkel, H. (2006). Estudios en Etnometodología. Barcelona: Anthropos/ UNAM.

[11] López, M.; Figueroa, C. y Rajland, B.(Ed.). (2010). Temas y procesos de la historia reciente de América Latina. Santiago de Chile: CLASCO/Arcis. Recuperado de: http://biblioteca.clacso.edu.ar/clacso/coediciones/20100827084236/hist oriarec.pdf

[12] MEI (2006). Modelo Educativo Institucional. Universidad Autónoma de Aguascalientes. Recuperado de https://www.uaa.mx/principal/pdf/modeloeducativo.pdf

[13] PEED (2020). Programa Emergente de Educación a Distancia. Boletín 144. Universidad Autónoma de Aguascalientes. Recuperado de: https://www.uaa.mx/portal/pdf-entradas/?post id=17418

[14] Soto-Gamboa, A. (2004). Historia del presente: esta do de la cuestión y conceptualización. En Historia Actual Online, 3, pp. 101-116. Asociación de Historia Actual. Área de Historia Contemporánea. Cádiz: Facultad de Filosofía y Letras, Universidad de Cádiz. Recuperado de: https://www.historiaactual.org/Publicaciones/index.php/hao/article/view/34 